\title{
Rosmarinus officinalis L. leaf extract improves Anxity impairment induced by tropane alkaloids extracted from Datura stramonium
} Latifa Didou ${ }^{1}$, Fatima-Zahra Azzaoui ${ }^{1}$, Ahmed omar touhami Ahami ${ }^{1}$,Soumia Ed-Day ${ }^{1},{\text { Fatima Ezzahra } \text { Kacimi }^{2},}^{\text {Samira } \text { Boulbaroud }}{ }^{2}$.

\footnotetext{
${ }^{1}$ Biology and Health laboratory,Department of Biology, Faculty of Science, Ibn Tofail University, PoBox.133, Kenitra, Morocco.

${ }^{2}$ Polydisciplinary Faculty, Sultan Moulay Slimane University, Beni Mellal, Morocco.
}

\begin{abstract}
The alkaloid extract of datura stramonium (rich of atropine, hyoscyamine, scopolamine) is used to evaluate its effect on the central nervous system in rats (locomotion, anxiety). The animals received the alkaloid extract of datura by intraperitoneal injection. However, after the injection a group of rats got an inhibitory treatment contain flavonoids extracted from Rosmarinus officinalis. At the dose of $50 \mathrm{mg} / \mathrm{kg} /$ body weight of the alkaloid extract, the female rats have shown a reduction of locomotor activity and an induction of an anxiogenic effect. In the other hand, the mixture of Datura stramonium and Rosmarinus officinalis showed a neuroprotective action with regard to the disorders induced by extracts of Datura stramonium.
\end{abstract}

\section{Introduction}

Datura stramonium is a wild plant that belongs to Solanaceae family. This later is known by its hallucinogenic uses and its effects on memory. This plant has several names, because of its properties, such as, crazy grass, devil's grass. All parts of datura are toxic and contain tropanic alkaloids: L-hyoscyamine, atropine and scopolamine. The quantities of alkaloids and their proportions are related to the species, the part of the plant and the time of year [1]. This plant has numerous uses; for example, in Europe it is used for therapeutic purposes for example as antiasthmatic, but in Africa and Asia it is used for magical properties, and due to its hallucinogenic virtues, Datura stramonium is currently used by drug addicts. [1].

Flavonoids are a large group of biological active compounds that could be found in our food, especially vegetables and fruits, and often have beneficial effects on the body. Plants belonging to the Lamiaceae family are known for their activity against various chronic disease, such as atherosclerosis (stroke) and neurodegenerative disease through their phenolic compounds [2].

\section{Materials and Methods}

\subsection{Preparation of Extract}

\subsubsection{Total alkaloids extract}

The isolation of total alkaloids from Datura stramonium seeds are obtained by liquid-liquid extraction, based on the difference in solubility of alkaloids in acid and alkaline medium according to the methods described by $[3,4]$.The extract collected and condensed subsequently using a rotavapor.

\subsubsection{Flavonoids Extraction}

A quantity ( $\mathrm{m}=10 \mathrm{~g}$ ) of Rosmarinus officinale powder was put in $320 \mathrm{ml}$ of $\mathrm{HCl}(2 \mathrm{~N})$ hydrochloric acid which was placed in a water bath at $100{ }^{\circ} \mathrm{C}$. for 40 minutes. The extracts were then filtered through a filter paper by vacuum filtration, the aqueous phase neutralized and small amounts of saturated water added 
to NAOH. The organic phase is dried by filtration on filter paper supporting anhydrous sodium sulphate.

The recovered phase undergoes clashes in a separatory funnel with ethyl acetate. The ethyl acetate phases recovered are evaporated under vacuum at $65^{\circ} \mathrm{C}$ in a rotavapor.

\subsection{Animals:}

Female rats of Wistar strain aged 3 months and weighing $(160 \pm 40) \mathrm{g}$ are used in this study. The animals are bred at the Department of Life Sciences, Faculty of Sciences, Kenitra, Morocco, at an ambient temperature of $25 \pm 4^{\circ} \mathrm{C}$. The rats were divided into 3 groups: Groups 1: groups of control animals received saline water. Groups 2: Groups of animals that received total alkaloids from Datura stramonium $L$ at a dose of 50 $\mathrm{mg} / \mathrm{kg}$. Groups 3: groups of animals that received a mixture of total alkaloids from Datura stramonium $L+$ seeds of the total flavonoids of Rosmarinus officinalis extract at a dose of $50 \mathrm{mg} / \mathrm{kg}$. The three groups of rats were exposed to treatment daily for 30 days by intraperitoneal injections.

\subsection{Behavioral assessment}

\subsubsection{Behavioral Tests Open-Field Test (OF)}

To assess possible effects of the plant extract on locomotor activity and the ability to response to novel environment, the rats were evaluated in open-field test during 10min. Apparatus consisted of an open wooden bow $(100 \times 100 \times 40)$ covered by a white consistent plastic. The floor of the field is divided into 25 squares unit by black lines and lit in the center with halogen lamps of $60 \mathrm{~W}$ installed in the ceiling[5,6]. The frequencies of the squares crossed by the animals (locomotion) and number of rearing in exploratory activity (anxiety level), were recorded by video camera positioned above the open field.

\subsubsection{Novel Object Recognition Task (NOR)}

The test and its procedures are described by several authors $[7,8]$ The object recognition test is particularly useful for studying declarative memory in rodents because it uses their natural preference for exploring a new object instead of the familiar object. This task evaluates the ability of rats to recognize a new object in a known environment.

Control animals typically spend more time exploring the new object, reflecting the use of memory and learning processes. The absence of difference in the exploration of objects can be interpreted as a memory deficit. The apparatus for this test consists of an open wooden enclosure consisting of an opaque square horizontal floor of black color $40 \mathrm{~cm}$ square and black vertical walls of a height of $50 \mathrm{~cm}$.

After each session, the objects are soaked in alcohol (96\% ethanol), then dried to eliminate as much as possible the odorous traces left by the animals. For the same reason, two different sets of identical objects are used alternately for the acquisition and retention sessions. The animals are first subjected to a session of habituation: for the task of object recognition, the rat is always placed in the middle of one side of the enclosure, the head directed towards the wall. The duration of the habituation session was $5 \mathrm{~min}$. Sessions of acquisition and retention of the task of recognition of object The day after the session of habituation, the animal is deposited at the same place as the day before and explores freely during $5 \mathrm{~min}$ the enclosure where was placed an object AT. After a so-called "retention" period of $(2 \mathrm{H})$, the animal is placed in the chamber for 5 minutes and freely explores the two objects (A and B).

\subsection{Statistical analysis}

In order to constitute the study matrix, the data was entered to Excel. And due to the non-normality of the sample distribution and the inequality of variances, nonparametric statistical tests were used. The MannWhitney test to compare groups 2 to 2 was used to detect significant differences between the groups studied. Statistical processing was performed using the Statistica software. The results were expressed as mean \pm standard mean error (ESM). The differences were considered significant for values of $\mathrm{p}<0.05$.

\section{Results}

\subsection{Behavioral assessment}

\subsubsection{Locomotor activity}

There was a significant decrease in the number of cells visited (NTC) in the group treated with the total alkaloids extracted from the seeds of Datura stramonium $L$. compared to the control rats. The same result was observed between the alkaloid group and the rats received the mixture of extracts of Datura stramonium (total alkaloids) and Rosmarinus officinalis (total flavonoids).

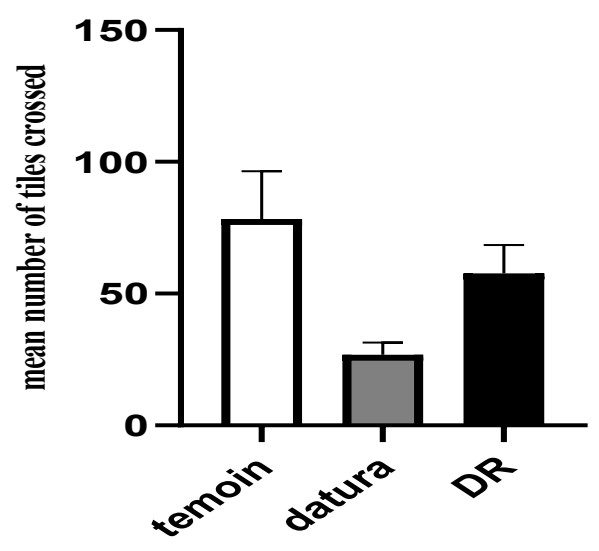


Fig. 1:Effects of extracts of Datura stramonium (total alkaloids) and Rosmarinus officinalis (total flavonoids) administered by IP at a dose of $50 \mathrm{mg} / \mathrm{kg}$ on the locomotor activity of experimental rats in the Open Field test. The significance level is $0.05 .{ }^{*} \mathrm{p}<0.05,{ }^{* *} \mathrm{p}<0.01,{ }^{* * *} \mathrm{p}<0.001$.

\subsubsection{Level of anxiety measured in OF}

The behavior of the rats in OF shows that the injection of Datura stramonium induces anxiety the group treated less frequently explores the central area in comparison with the control group $(p<0.05)$. On the other hand, the group treated with the mixture of extracts Datura stramonium (total alkaloids) and Rosmarinus officinalis (total flavonoids) showed a rather important exploration compared to the group treated with Datura ( $\mathrm{p}$ $<0.05)$.

In addition, the group treated with Datura stramonium extract (total alkaloids) spends less time in the core area (TPC) than the control group $(p<0.05)$ than the control group $(p<0.05)$. On the other hand, the group treated with the mixture of extracts of the two plants (DR) spends more time in the central zone than the group treated with the alkaloids $(\mathrm{p}<0.05)$.
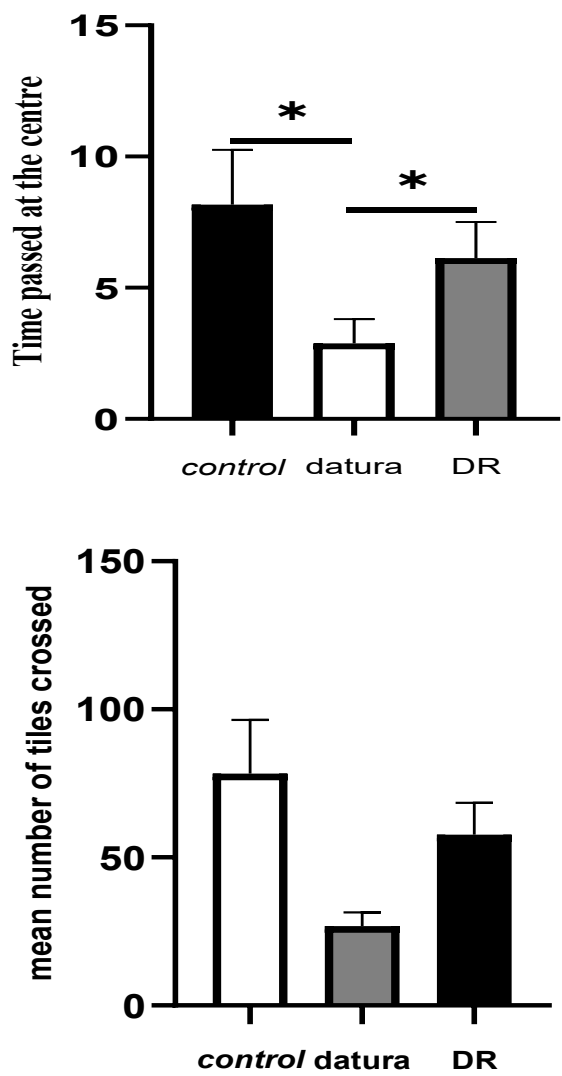

Fig. 2: Effects of extracts of Datura stramonium (total alkaloids) and Rosmarinus officinalis (total flavonoids) administered by IP at the dose of $50 \mathrm{mg} / \mathrm{kg}$ on the number of central cells visited (NCC) (A) the time spent in the center ( TCP) evaluated by the Open Field test.(B) Number of center

littles visited. The results have represented on average by the number of tiles visited \pm Standard Mean Error (SEM). * Significant difference between the different groups (Witness versus Datura and datura versus DR); $p<0.05$ by Mann Whitney U-test.

\subsubsection{Object recognition memory}

Effect on short-term memory: Figure 3 shows that the injection Datura stramonium does not induce any significant difference in the time taken to explore the two objects during the acquisition session. During the retention session, the control rats were able to differentiate between the two objects (familiar and new object). On the other hand, our results showed that the rats received the alkaloid extracts of Datura stramonium explore the familiar object and the new object in the same way, this exploration remains significantly weak compared to the control group $(\mathrm{p}<0.001)$ and DR ( $\mathrm{p}$ $<0.01)$ ). The results also showed non-significant rest of the index of recognition of the new object in the groups studied compared to the control group. However, no significant difference between the group receiving alkaloid extracts of Datura stramonium and the group receives the mixture DR
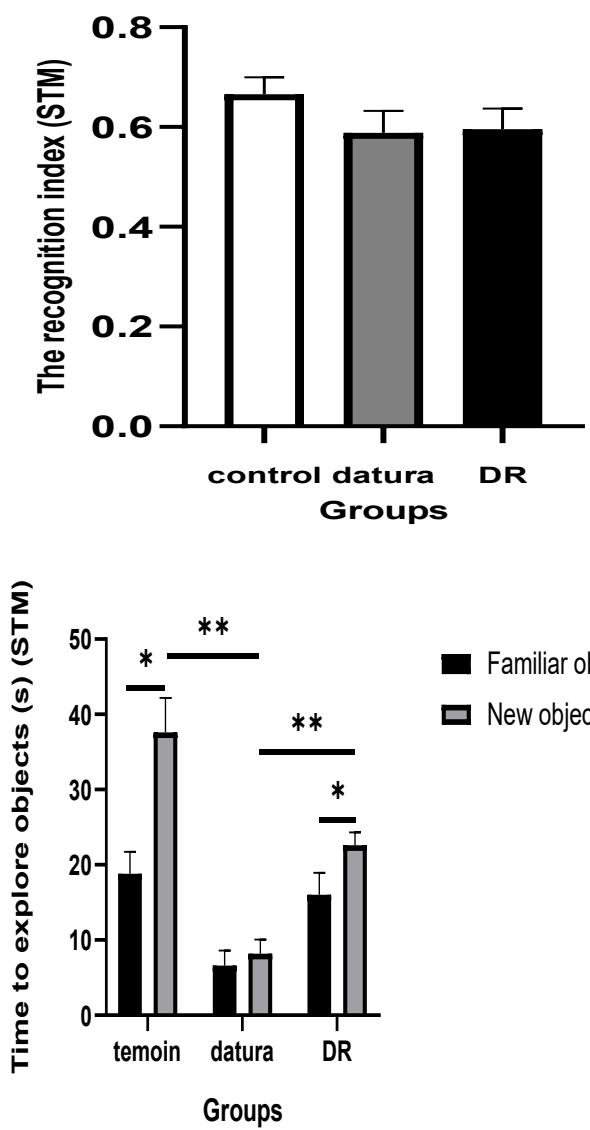

Familiar object exploration time

口 New object exploration time

Fig. 3Effects of extracts of Datura stramonium (total alkaloids) and Rosmarinus officinalis (total flavonoids) administered by IP at the dose of $50 \mathrm{mg} / \mathrm{kgA}$. The recognition index at $2 \mathrm{~h}$ after training (STM)B. timeto explore the objects. The results are expressed as Mean \pm SEM. The significance level is 0.05 . ${ }^{*} \mathrm{p}<$ $0.05,{ }^{* *} \mathrm{p}<0.01,{ }^{* * *} \mathrm{p}<0.001$ ( One Way ANOVA and post hoc multiple comparisons).

Effect on long-term memory:All groups explore the two objects in equivalence during the acquisition 
session. During the retention session, the rats were able to differentiate between the two objects (familiar and new object). On the other hand, our results showed that the rats receiving the alkaloid extracts of Datura stramonium spent less time in the exploration of the new object compared to the control group $(\mathrm{P}<0.001)$. Similar to the control group, this group significantly explores the new object than the DR group $(\mathrm{p}<0.01)$. While no significant variation in the exploration of the new object has been reported between the control group and the DR group. The index of recognition of the new object is significantly high in the group treated with Datura extracts compared to the group, the same result was observed in the DR group, however the DR group similarly explores the new object at the same time. The detention session.
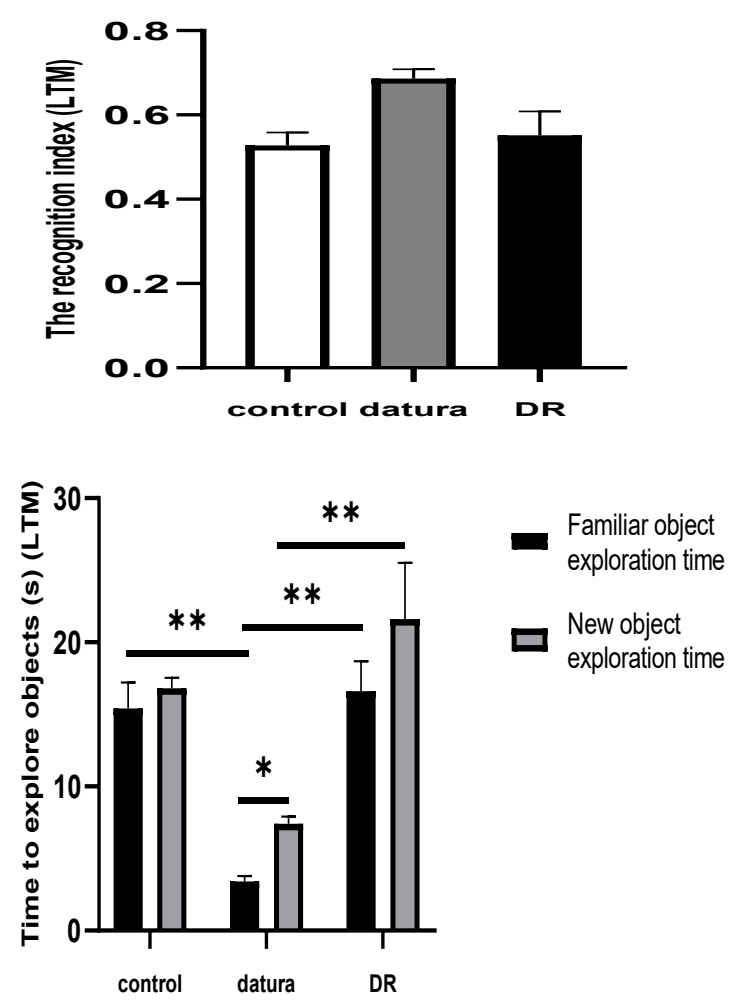

Fig. 4Effects of extracts of Datura stramonium (total alkaloids) and Rosmarinus officinalis (total flavonoids) administered by IP at the dose of $50 \mathrm{mg} / \mathrm{kgA}$.The recognition index at $24 \mathrm{~h}$ after training(LTM)B.Timeto explore the objects. The results are expressed as Mean \pm SEM. The significance level is 0.05 . $* \mathrm{p}<0.05, * * \mathrm{p}<0.01,{ }^{* *} \mathrm{p}<0.001$ ( One Way ANOVA and post hoc multiple comparisons).

\section{Discussion}

In our experiment, we observed a fairly significant increase in the absolute or relative weights of organs, especially the liver, in the alkaloid-treated group. Through this result, we noticed a significant similarity with the results of Duggan et al. (1989) [9]. Where they have found in turn, a significant increase in the relative weight of the liver, brain, heart and spleen. In contrast to our study, the other organs did not show any significant difference in weight either in rats injected with alkaloids or flavonoids.

After analyzing the performance of the animal in the behavioral tests, we observed a significant decrease in motor activity, and the effect of anxiety according to NTC, NCC and TPC parameter's in female rats injected with alkaloids, and it could be related to the decrease in motor activity as a result of the sedative effect of the extract on the nervous system. Through these results we conclude that alkaloids affect locomotor activity and cause an alarming effect in rats, our results is similar to Elisabet sky and Costa-Campos's results (2006) [10] which demonstrated that the alkaloids present in the extract of Datura would originally have a sedative effect. Moreover, no change in spontaneous locomotor activity was detected, and anxiety effects were not observed in rats that were injected with alkaloids followed by flavonoids, so the results could be explained by thye fact that flavonoids reduce anxiety symptoms caused by alkaloids [11]

Control rats didn't show any decrease in amnesia, in fact, all animal spend more time $( \pm 10 \mathrm{~s})$ in exploring the new object during the recognition session. In addition, female rats treated with Datura seed extract spent a lot of time exploring new as well as old objects, however their cholinergic synaptic transmission during the training session, is still inhibited by scopolamine and therefore they could not memorize the two objects presented. These results are in complete agreement with the results of Nikolaus (2006) and Nikolaus (2007) who demonstrated that mnemic deficit caused by scopolamine through the object recognition test, as well as to the results of Luttgen (2005), Naghdi (2006) and Ben-Barak and Dodai (1980) who found that Sub-chronic administration of scopolamine increases the number of muscarinic receptors in the rat hippocampus. Additionally, it has been observed that rats injected with flavonoids exhibit an exploration of the new object in the object recognition test equivalent to that of control rats $[12,13,14,15]$.

The same thing was shown by the results of González et al. 2007, which confirmed that Rosmarinus Officinalis extract $(150$ and $300 \mathrm{mg} / \mathrm{kg}$ ) improves the learning processes and memory of mice, and therefore we conclude that Datura stramonium extract significantly reduces retention in the three educational situations used in this study [16].

\section{Conclusion}

The goal of this study is to evaluate the effect of alkaloid extracts of Datura stramonium on neurocognitive disorders such as memory, locomotion and anxiety assessed through object recognition memory tests and by open field testing is. A preventive treatment of these disorders by natural molecules (flavonoids) extracted from the plant Rosmarinus officinalis was administered to rats having received an alkaloid extract from Datura. The results obtained showed that the IP injection of the extracts of Datura stramonium seeds into adult female Wistar rats induces an anxiogenic effect 
associated with a significant alteration of the spontaneous locomotor activity, also induces an impairment of the memory. In addition, the administration of flavonoid extracts showed an improvement in cognitive performance in the rats that received the mixture of the two extracts. It seems that the activity of Rosmarinus officinalis represents a possible track for the preventive treatment against the risks of certain neurodegenerative diseases.

\section{References}

1. E. Miraldi, A. Masti, S. Ferri, I.B. Comparini, Distribution of hyoscyamine and scopolamine in Datura stramonium, Fitoterapia, 72, 644-648 (2001)

2. F. Fahim, A. Esmat, H. Fadel \& K. Hassan, Allied studies on the effect of Rosmarinus officinalis $L$. on experimental hepatotoxicity and mutagenesis, IJoFSci. and Nut., 50:6, 413427(1999)

3. PA. Steenkamp, NM. Harding, FR .van Heerden, BE. van Wyk. Fatal Datura poisoning: identification of atropine and scopolamine by high performance liquid chromatography/photodiode array/mass spectrometry. Forensic Sci Int.;145(1):31-9(2004)

4. E. Miraldi, A. Masti, S. Ferri \& C.I. Barni "Distribution of hyoscyamine and scopolamine in Datura stramonium, Fitoterapia, 72, 644-648 (2001)

5. FZ. Azzaoui, AOT. Ahami and A. Khadmaoui: Impact of aluminum sub-chronic toxicity on body weight and recognition memory of wistar rat. Pakistan Journal of Biological Sci.; 11 : 1830-1834(2008)

6. FZ. Azzaoui, AOT. Ahami and A. Khadmaoui: Impact of lead sub-chronic toxicity on recognition memory and motor activity of Wistar rat. Pak. Pakistan Journal of Biological Sciences; 12: $173-$ 177(2009)

7. A. Ennaceur, J. Delacour, A new one-trial test for neurobiological studies of memory in rats. 1. Behavioral data. BehavBrainRes, 31,47-59. (1988).

8. F.Z. Azzaoui,. Le plomb et l'aluminium: Etude d'impact sur la santé et évaluation du potentiel chélateur de certaines plantes médicinales. Thèse. Université Ibn Tofail,Kenitra, Maroc. (2009)

9. G. M. Dugan, M. R. Gumbmann and M. Friedman, Toxicological evaluation of jimson weed (Datura stramonium) seed. Food and Chemical Toxicology 27, 501-510(1972)

10. J. M. Gowdy, Stramonium intoxication. Journal of the American Medical Association, 221, 585587(1989)

11. E. Elisabetsky, L. Costa-Campos, The alkaloid alstonine: a review of its pharmacological properties. Evid Based Complement Alternat Med.;3(1):39-48(2006)

12. Su. Kang-Yi et al. "Rutin, a flavonoid and principal component of saussurea involucrata, attenuates physical fatigue in a forced swimming mouse model." International journal of medical sciences vol. 11,5; 528-37(29 Mar. 2014)

13. , M. Lüttgen, E. Elvander, N. Madjid, \& S. O. Ögren. Analysis of the role of 5-HT1A receptors in spatial and aversive learning in the rat. Neuropharmacology, 48(6), 830-852(2005)

14. N. Naghdi, , M. Rezaei, \&Y. Fathollahi, Microinjection of ritanserin into the CAl region of hippocampus improves scopolamine-induced amnesia in adult male rats. Behavioural Brain Research, 168(2), 215-220((2006)

15. J. Ben-Barak, \&Y. Dudai, Scopolamine induces an increase in muscarinic receptor level in rat hippocampus. Brain Research, 193(1), 309313(1980).

16. T.M.E. González; E.I. Peña; A.L. Martínez; , J.P. Moreno; P.G. Fefer; M.D. Campos; F.J.L. Muñoz, Evaluation of the antinociceptive effect of Rosmarinus officinalis L. using three different experimental models in rodents. $\mathrm{J}$.

Ethnopharmacol., v. 111, p.476-482(2007) 